\title{
La comunicación científica en América Latina es abierta, colaborativa y no comercial. Desafíos para las revistas
}

Scientific communication in Latin America is open, collaborative and non-commercial. Challenges for journals

\author{
Dominique Babini \\ Consejo Latinoamericano de Ciencias Sociales (CLACSO), Argentina \\ dasbabini@gmail.com
}

\begin{abstract}
Resumen:
América Latina, en donde la investigación y la difusión de los resultados se financian principalmente con fondos públicos, es la región del mundo con mayor adopción del sistema de acceso abierto a los contenidos en revistas científicas. Se destacan iniciativas regionales surgidas en el ámbito académico tales como Latindex, SciELO y Redalyc, que se complementan con portales nacionales e institucionales de revistas de acceso abierto, todas ellas iniciativas colaborativas sin fines comerciales. En base a la experiencia de CLACSO en la región y a su participación en los debates internacionales sobre el futuro de las comunicaciones científicas, en este artículo se comparten reflexiones sobre algunos de los desafíos que se plantean a este modelo colaborativo de acceso abierto en América Latina. Por ejemplo: los nuevos requerimientos que surgen desde la ciencia abierta, la necesidad de contar con indicadores de acceso abierto para que los procesos de evaluación puedan valorar esa producción publicada en revistas de la región y la urgencia de fortalecer la gestión regional colaborativa del acceso abierto y sus indicadores, sin fines comerciales.

Palabras clave: Comunicaciones científicas, Revistas científicas, Acceso abierto, Ciencia abierta, Indicadores de evaluación, Evaluación de la investigación.
\end{abstract}

\section{Abstract:}

Latin America, where research and journals that publish research results, are both activities that are mainly publicly-funded, is the region of the world with the greatest adoption of open access, to scientific journals, highlighting scholarly-led regional initiatives such as Latindex, SciELO, Redalyc, which are complemented by national and institutional open access journal portals, all collaborative non-commercial initiatives. In this article, and based on CLACSO's experience in the region and its participation in international debates on the future of open access scholarly communications, views are shared on some of the challenges that this collaborative open access model poses to journals published in countries of Latin America. As is the case of open science requirements for journals, the need to have open access indicators so that evaluation processes can assess the contributions from research output published in journals within the region, and the urgency to strengthen collaborative non-commercial models to manage open access and its indicators.

KEYWORDS: Scientific communications, Journals, Open access, Open science, Evaluation indicators, Research evaluation.

\section{INTRODUCCIÓN ${ }^{1}$}

En América Latina, tanto la investigación científica como la comunicación de sus resultados son actividades financiadas principalmente con fondos públicos; es decir que la participación estatal es protagonista en la actividad científica y en las comunicaciones científicas en la región. Tres cuartas partes de los investigadores latinoamericanos están radicados en las universidades, principalmente en las universidades públicas (Albornoz, Barrere y Sokil, 2017; OCTS, 2018), y es en el mismo espacio universitario en donde se publican la mayoría de las revistas (Salatino, 2017), publicaciones con alcances geográficos muchas veces locales y regionales (Vessuri, Guédon y Cetto, 2013; Salatino 2018). En mayor medida que en otras regiones del mundo, las revistas científicas y académicas están utilizando modelos de publicación en acceso abierto, que surgen tanto del sentido de misión pública de la universidad latinoamericana como del consenso acerca de su efectividad para compartir conocimiento (Alperin, Fischman y Willinsky, 2012). Además, las

Recepción: 20 de octubre de 2018 | Aceptación: 28 de marzo de 2019 | Publicación: 25 de abril de 2019 
diversas modalidades de acceso abierto son financiadas principalmente con fondos públicos destinados a la investigación (UNESCO-GOAP, s.f.).

Para aquellas revistas en acceso abierto que tienen un porcentaje alto de contenidos originales de investigación y procesos de revisión por pares, se presenta la oportunidad de postularse para ser aceptadas en los servicios regionales de indización como Latindex Catálogo, SciELO y Redalyc, que multiplican su visibilidad. Además, estas revistas pueden postularse a DOAJ (Directory of Open Access Journals). Tanto SciELO como Redalyc brindan indicadores para procesos de evaluación, los cuales se describen en un libro publicado por el Consejo Latinoamericano de Ciencias Sociales (CLACSO) el cual se comenta más adelante en este artículo. Estos sistemas regionales de revistas de calidad (Latindex-Catálogo, SciELO y Redalyc) "elevaron el perfil así como la calidad de las revistas académicas en América Latina" (Alperin, Fischman y Willinsky, 2012, p. 23). Además, debe destacarse que el trabajo de los portales regionales se realiza de manera organizada, metodológica y cooperativa (Cetto, Alonso Gamboa, Packer y Aguado López, 2015).

El caso de la gestión del acceso abierto en modalidades colaborativas y cooperativas en América Latina es un ejemplo seguido con gran interés por la comunidad internacional. No hay evento internacional sobre comunicaciones académicas en el que CLACSO esté invitado en donde no se pregunte cómo se logró, en nuestra región, mantener la gestión de la edición de publicaciones y el acceso abierto dentro de la comunidad académica. Hace muchas décadas que en el Norte, en general, las comunicaciones académicas se tercerizaron al sector comercial, una industria que hoy está concentrada en unas pocas empresas que manejan los precios para asegurar a los accionistas una ganancia del $30 \mathrm{al} 40 \%$ anual, lo que la constituye en una de las industrias más rentables del mundo (Larivière, Haustein y Mongeon 2015; Buranyi, 2017), que funciona restando ese plus de ganancia de los presupuestos de investigación de los distintos países. Ahora que el modelo de acceso abierto avanza en el mundo, la industria editorial comercial internacional capta, además del modelo de suscripciones a revistas que exigen pagar para leer, el negocio que ellos mismos ven en el acceso abierto, cobrando en este caso por publicar en abierto a medida que avanzan las políticas y legislaciones que exigen difundir sin cobrar por leer. Pretenden, por supuesto, seguir cobrando, si es posible, en ambos negocios. Esto ha generado críticas y reacciones del sector académico y científico, y es muy interesante observar cómo en Estados Unidos, Canadá y Europa están ahora muy tímidamente surgiendo propuestas de modelos cooperativos y colaborativos para gestionar la publicación de revistas desde las mismas comunidades académicas, sin intervención comercial.

Nuestra fortaleza en América Latina es que estamos donde ellos quieren llegar: tenemos comunicaciones científicas gestionadas por la misma comunidad científica, en formas colaborativas, sin tercerización comercial. Por este motivo consideramos que es necesario fortalecer los modelos colaborativos que ya tenemos en la región y crear alianzas con los nuevos modelos cooperativos que se están iniciando en el Norte, para ir hacia un acceso abierto global no comercial. Recorriendo ese camino, compartimos aquí reflexiones sobre algunos desafíos en el caso de las revistas de la región, su valoración y gestión.

\section{EN UN CONTEXTO DE CIENCIA ABIERTA, LAS REVISTAS CIENTÍFICAS INTERACTÚAN CON UNA DIVERSIDAD DE OBJETOS DIGITALES DE INVESTIGACIÓN}

En las comunicaciones científicas y académicas en entornos digitales y en procesos de ciencia abierta no sólo incorporamos a nuestras plataformas las revistas y los artículos con sus metadatos, también tenemos que incorporar enlaces a una diversidad de objetos digitales que se han ido creando durante el proceso de investigación y han sido depositados en plataformas y repositorios que les asignan identificadores para enlazar a ellos cuando se los menciona en los artículos de las revistas. Por ejemplo, desde el mismo artículo se pueden brindar enlaces a informes de avances de investigación publicados en un repositorio de preprints antes de que apareciera el artículo para tener comentarios que permitan mejorar el texto; a sets de datos o bases de datos generados durante la investigación y almacenados en repositorios digitales; a aplicaciones de software 
desarrolladas para ese trabajo específico y archivadas también en un repositorio digital; a videos (alojados en Youtube o en otros repositorios) que sirven para documentar la descripción del proyecto o en donde se muestran entrevistas que autorizan su difusión; a enlaces a los textos completos de las citas y referencias bibliográficas del artículo cuando estén disponibles en acceso abierto.

Además de manejar el texto del artículo, sus imágenes, tablas y gráficos, ahora el proceso editorial y el proceso de difusión del artículo en diversas plataformas debe manejar también los enlaces desde el artículo a una diversidad de objetos digitales que, cuando se trabaja en ciencia abierta, son el resultado de procesos continuos de comunicación que permiten mayor participación en la producción y comunicación de los nuevos conocimientos, en tanto el artículo es sólo uno de los tantos productos de comunicación. Debemos facilitar el trabajo para que las computadoras y los buscadores dialoguen entre sí, con el fin de que ese objeto digital -artículo- tenga posibilidad de adquirir visibilidad y acceso en conversaciones internacionales de la ciencia, lo que permitirá también la inserción de nuestros investigadores e investigadoras a nivel internacional. Para ello, los objetos digitales de la comunicación científica deben ser lo más completos posibles en sí mismos: incluir el contenido, citas abiertas (estructuradas y legibles por computadora) y metadatos estandarizados según normas internacionales para evitar encontrarnos con textos en PDF sobre los cuales no sabemos si se trata de un artículo de una revista, un capítulo de un libro, o con sets de datos sueltos sin información sobre la investigación que los incluye. Lo ideal es que cada objeto digital informe también si el contenido tuvo revisión por pares y qué tipo de revisión por pares se aplicó.

\section{INDICADORES DE ACCESO ABIERTO COMO CONTRIBUCIÓN A LOS PROCESOS DE EVALUACIÓN}

Si queremos que en los procesos de evaluación en América Latina se valore también la producción publicada en acceso abierto en revistas de la región y al mismo tiempo ser inclusivos para abarcar una diversidad de publicaciones -y no sólo aquellas que son aceptadas por los servicios de indizaciones internacionales o regionales-, debemos trabajar juntos en el desarrollo de indicadores complementarios que muestren qué circulación, uso e impacto tienen los objetos digitales que publicamos en diversos formatos y en plataformas institucionales, nacionales y regionales.

Esos indicadores, además, deben estar inspirados en las recomendaciones de las principales declaraciones internacionales. Dos de las principales declaraciones que nos pueden inspirar en los procesos de evaluación y en el desarrollo de indicadores son las declaraciones DORA (Declaración de San Francisco de Evaluación de la Investigación) (2012) y los principios del Leiden Manifesto for Research Metrics (2015). En CLACSO tenemos preocupación por la falta de actualización de muchos de los sistemas de evaluación en la región respecto a estas nuevas tendencias internacionales, que no son tomadas en cuenta. Por ejemplo, la recomendación de no utilizar métricas basadas en revistas, tales como el índice de impacto de las mismas, como una medida sustitutiva para el análisis de la calidad de los artículos individuales, como recomienda DORA (2012).

Tal como se los describe en un libro publicado por CLACSO (Alperin, Babini y Fischman 2014), ya se dispone en la región de los primeros indicadores de acceso abierto que pueden utilizarse en procesos de evaluación, como son los casos de los indicadores de SciELO y Redalyc, así como el del repositorio temático de CLACSO, para sumar a los indicadores tradicionales que se utilizan en nuestra región, que son principalmente los de WoS y Scopus. Luego de la publicación de este libro, la novedad es que SciELO acordó realizar el SciELO Citation Index con la empresa internacional Clarivate que ofrece - pago mediante- acceso a informes de uso e impacto de la producción científica a los capítulos locales de la red SciELO. Este es el tipo de desarrollos que desde CLACSO desearíamos se hicieran en forma colaborativa entre universidades e instituciones de la región, en las que hay gran capacidad informática y académica para lograr modelos no comerciales. Como sucede en el caso de Redalyc, que en el ámbito de la Universidad Autónoma del Estado de México (UAEM) desarrolló sus indicadores sobre 1260 revistas y ahora inicia un proceso de 
descentralización con el proyecto AMELI en colaboración con CLACSO y un conjunto de universidades de la región.

\section{CRECIMIENTO Y SUSTENTABILIDAD DE LAS REVISTAS CIENTÍFICAS Y ACADÉMICAS EN ACCESO ABIERTO EN AMÉRicA LATINA Y SU INDEXACión CON MODElOS COLABORATIVOS No comerciales. Protagonismo de las universidades}

El tercer desafío que queremos compartir desde CLACSO es la necesidad de seguir creciendo en modalidades colaborativas y cooperativas, para que siga siendo sustentable el crecimiento y los nuevos desarrollos del modelo latinoamericano sin que tengamos que pasar a opciones comerciales que, como ya lo vivimos con el caso de las suscripciones a revistas internacionales, no defienden los intereses de la academia sino los del mercado.

Como dice Jean-Claude Guédon (2018), la comercialización de las revistas en el Norte fue una contrarrevolución ya que esto fue cambiando la naturaleza de las revistas, que se transformaron en mercancía del mercado. En el Norte se pasó de cobrar por leer a cobrar por publicar en acceso abierto. A partir de esto, la división no será entre quienes pueden o no acceder a las publicaciones científicas (por el costo de las suscripciones o por el pago de artículos individuales), sino entre quienes puedan o no pagar el costo por publicar en acceso abierto, ya sea por artículo publicado (en inglés APC: Article Processing Charges) o por libro publicado (BPC: Book Processing Charges). "El precio se basa en cuánto dinero asumen que tienes. Cobran lo que aguante el mercado", dice John Willinsky de la Universidad de Stanford -fundador de PKP- en una entrevista publicada en el diario El País de España (Willinsky, 2018). Bianca Kramer (2018), investigadora de la Universidad de Utrecht en los Países Bajos, propone formularnos las siguientes preguntas:

¿El dinero ganado por los APC fluye de nuevo a los editores del Norte, o vuelve al Sur y se invierte en la investigación local y la infraestructura de publicación / difusión local?

¿El sistema favorece la investigación patrocinada con financiamiento del Norte (ya sea directamente o mediante la colaboración) al facilitar la publicación de esa investigación en acceso abierto, a expensas de otras investigaciones locales?

El sistema de publicación en acceso abierto en revistas de corriente principal, ¿impone un pensamiento basado en el factor de impacto de las revistas que impulsan ciertos tipos de investigación, a expensas de otros tipos de investigación que podrían ser más relevantes a nivel local?

En la última reunión del grupo de consorcios de Iberoamérica que compran a nivel nacional las suscripciones a las revistas científicas internacionales, se acordó que el modelo de cobrar por publicar en acceso abierto no es viable en la región, y se recomendó a las instituciones no generar fondos para publicar en revistas que siguen ese modelo (Consorcios de Iberoamérica y el Caribe. Declaración de la Primera Reunión, 2017).

Con el modelo de cobrar por publicar cada vez más fondos de investigación están siendo transferidos a los accionistas de editoriales comerciales internacionales, cuando, según el directorio internacional DOAJ, el $65 \%$ de las revistas científicas y académicas de calidad del mundo que se publican en acceso abierto no cobran por publicar.

Para incentivar la publicación en revistas que no estén en el circuito comercial, necesitamos que las agencias de evaluación valoren la calidad de los artículos que los investigadores y las instituciones publican y no que sigan utilizando indicadores de revista como el Factor de Impacto, según recomienda DORA (2012).

Desde CLACSO recomendamos alejarnos del camino comercial fortaleciendo los sistemas colaborativos y cooperativos como es tradición en la región y como se está experimentando también en el Norte para evitar el abuso comercial. En América Latina hemos demostrado que tenemos las condiciones para ir por un camino diferente en acceso abierto y no caer en manos de los editores comerciales internacionales (Cetto, 2018). 
Por esto, CLACSO publicó su declaración sobre el acceso abierto al conocimiento gestionado como un bien común (CLACSO, 2015), cuyos principios respaldan el acceso abierto de calidad y su gestión por parte de la comunidad científica y académica.

El abuso comercial puede llegar también a través de la reutilización comercial de contenidos que están en acceso abierto. En la región latinoamericana hay múltiples voces que consideramos que si la producción científica y académica en acceso abierto es financiada con fondos públicos no se debe permitir el lucro con esos contenidos, posición que entra en conflicto con las licencias CC-BY. A este respecto, Latindex, Redalyc, CLACSO e IBICT emitieron su posición con la Declaración de México, en la cual recomiendan usar CC BY-NC-SA: Atribución-No Comercial-Compartir Igual (Declaración de México..., 2018).

\section{A MODO DE CIERRE, UNA INVITACIÓN A LAS UNIVERSIDADES}

Las universidades de nuestra región han demostrado tener amplia capacidad para desarrollar modelos colaborativos innovadores. A modo de ejemplo, en lo que se refiere a portales regionales de revistas de calidad, podemos mencionar a la Universidad Nacional Autónoma de México (UNAM), que desarrolló Latindex; la Universidad Autómoma del Estado de México (UAEM), que desarrolló Redalyc; y SciELO, que es también un desarrollo iniciado en el ámbito de la información científica (BIREME-Biblioteca Regional de Medicina). Asimismo, son las universidades de la región las principales protagonistas en la creación de los repositorios digitales con producción propia, incluyendo las revistas. Como mencionamos anteriormente, estas iniciativas están comenzando a generar recursos para contribuir a que los contenidos publicados en acceso abierto en la región puedan tener indicadores de utilidad en los procesos de evaluación (Alperin, Babini y Fischman, 2014).

Es necesario que otras universidades se sumen para que se consolide una red de universidades que colaboren en la investigación e innovación en estos temas. Por su parte, los organismos nacionales de ciencia y tecnología pueden incrementar el apoyo financiero a nuevas iniciativas colaborativas y cooperativas, como lo están haciendo hoy en el caso de La Referencia y SciELO, servicios que permiten valorar la producción científica que ellos financian.

Desde CLACSO participamos en el proyecto AMELI, que busca desde Redalyc consolidar un conjunto de universidades e instituciones con los objetivos y actividades que permitirán fortalecer en la región el acceso abierto y sus indicadores en modalidades no comerciales.

\section{REFERENCIAS}

Albornoz, M., Barrere, R., y Sokil, J. (2017). Las universidades lideran la I+D en América Latina. En M. Albornoz y R. Barrere (Coord.), El Estado de la Ciencia. Principales Indicadores de Ciencia y Tecnología Iberoamericanos / Interamericanos 2017 (pp. 31-44). Buenos Aires: Argentina, RICYT y OCTS-OEI. Recuperado de http://ww w.ricyt.org/files/Estado\%20de\%20la\%20Ciencia\%202017/El_Estado_de_la_Ciencia_2017_Completo.pdf

Alperin, J. P., Babini, D., y Fischman, G. (Eds.) (2014). Indicadores de acceso abierto y comunicaciones académicas en América Latina. Buenos Aires: CLACSO. Recuperado en http://biblioteca.clacso.edu.ar/clacso/se/201412170 52547/Indicadores_de_acceso_abierto.pdf

Alperin, J. P., Fischman, G. E., y Willinsky, J. (2012). Scholarly communication strategies in Latin America's research intensive universities. Revista Educación Superior y Sociedad, 16(2). Recuperado de http://ess.iesalc.unesco.org .ve/ess3/index.php/ess/article/view/409

Kramer, B. (2018). Comunicación en Radical Open Access email discussion list (Abril 4, 2018). Recuperado de www.jiscmail.ac.uk/cgi-bin/webadmin?A2=RADICALOPENACCESS;49c3b7af.1804 
Buranyi, S. (2017, junio 27). Is the staggeringly profitable business of scientific publishing bad for science? The Guardian. Recuperado de https://www.theguardian.com/science/2017/jun/27/profitable-business-scientificpublishing-bad-for-science

Cetto, A. M., Alonso Gamboa, J.O., Packer, A., y Aguado López, E. (2015). Enfoque regional a la comunicación científica. En J. P. Alperin y G. Fischman (Eds.), Hecho en Latinoamérica: acceso abierto, revistas académicas e innovaciones regionales (pp. 19-42). Buenos Aires: CLACSO. Recuperado de http://biblioteca.clacso.edu.ar/cl acso/se/20150722110704/HechoEnLatinoamerica.pdf

Cetto, A. M. (2018). From open access to open science in the Latin American ecosystem. En ISSN Conference 2018. Ecole Nationale d'Administration, Francia. Recuperado de https://webcast.in2p3.fr/video/from-open-accessto-open-science-in-the-latin-american-ecosystem

CLACSO (2015). Declaración de la Asamblea General de CLACSO sobre el acceso abierto al conocimiento gestionado como un bien común. Medellín, Colombia, 9 de noviembre 2015. Recuperado de https://www.clacso.org.ar/conferencia2015/documentos/asamblea/declaraciones/4-Declaracion-de-CLA CSO-sobre\%20el-acceso-abierto-al-conocimiento-gestionado-como-un-bien-comun.pdf

Consorcios de Iberoamérica y el Caribe. Declaración de la Primera Reunión, Ciudad de Juárez, México, 31 de agosto y 1 de setiembre 2017. Recuperado de http://reuniondeconsorcios.conricyt.mx/index.php/primera-reunion/d eclaraciones/

Declaración de México a favor del ecosistema latinoamericano de acceso abierto no comercial (2018). Recuperado de htt p://www.accesoabiertoalyc.org/declaracion-mexico/

Declaración de San Francisco de Evaluación de la Investigación [DORA] (2012). Recuperado de https://sfdora.org/r ead/es/

Guédon, J-C. (2018). The history of open access and its meaning. En ISSN Conference 2018. Video recuperado en ht tps://webcast.in2p3.fr/video/the-history-of-the-open-access-and-its-meaning

Larivière, V., Haustein, S., y Mongeon, P. (2015). The Oligopoly of Academic Publishers in the Digital Era. PLoS ONE, 10(6), e0127502. https://doi.org/10.1371/journal.pone.0127502

Leiden Manifesto for Research Metrics (2015). Recuperado de http://www.leidenmanifesto.org/

Observatorio Iberoamericano de la Ciencia, la Tecnología y la Sociedad [OCTS] (2018). Las universidades, pilares de la ciencia y la tecnología en América Latina. Buenos Aires: OEI. Recuperado en http://www.ricyt.org/

Salatino, M. (2017). La estructura del espacio latinoamericano de revistas cientificas (Tesis doctoral). Universidad Nacional de Cuyo, Mendoza, Argentina. Recuperado de http://bdigital.uncu.edu.ar/objetos_digitales/10720/ salatino-estructuraespaciolatinoamericano-revistascientficas.pdf

Salatino, M. (2018). Más allá de la indexación: Circuitos de publicación de Ciencias Sociales en Argentina y Brasil. Dados, 61(1), 255-287. https://dx.doi.org/10.1590/001152582018152

UNESCO-GOAP (s.f.). Global Open Access Portal. Latin America and the Caribbean. Recuperado de http://www.unesco.org/new/en/communication-and-information/portals-and-platforms/goap/access-by-r egion/latin-america-and-the-caribbean/

Vessuri, H., Guédon, J-C., y Cetto, A. M.(2013). Excellence or quality? Impact of the current competition regime on science and scientific publishing in Latin America and its implications for development. Current Sociology, 62(5), 647-665. Recuperado de http://eprints.rclis.org/23682/

Willinsky, J. (2018, abril 26). El acceso al conocimiento científico es un derecho humano. El País. Recuperado de htt ps://elpais.com/elpais/2018/04/25/ciencia/1524672252_074648.html

\section{Notas}

1 Presentación invitada en el $3^{\circ}$ Congreso Internacional de Editores Redalyc "Construyendo el modelo de publicación académica del sur global”. Trujillo, Perú, 16-18 mayo 2018. http://congreso.redalyc.org/ocs/public/congresoEditores/ index.html 